\title{
Some Problems in Optimally Stable Lagrangian Differentiation
}

\author{
By Herbert E. Salzer
}

\begin{abstract}
In many practical problems in numerical differentiation of a function $f(x)$ that is known, observed, measured, or found experimentally to limited accuracy, the computing error is often much more significant than the truncating error. In numerical differentiation of the $n$-point Lagrangian interpolation polynomial, i.e., $f^{(k)}(x) \sim \Sigma_{i=1}^{n} L_{i}^{n(k)}(x) f\left(x_{i}\right)$, a criterion for optimal stability is minimization of $\Sigma_{i=1}^{n}\left|L_{i}^{n(k)}(x)\right|$. Let $L \equiv L\left(n, k, x_{1}, \ldots, x_{n}\right.$; $x$ or $\left.x_{0}\right)=\Sigma_{i=1}^{n} \mid L_{i}^{n(k)}\left(x\right.$ or $\left.x_{0}\right) \mid$. For $x_{i}$ and fixed $x=x_{0}$ in $[-1,1]$, one problem is to find the $n x_{i}$ 's to give $L_{0} \equiv L_{0}\left(n, k, x_{0}\right)=\min L$. When the truncation error is negligible for any $x_{0}$ within $[-1,1]$, a second problem is to find $x_{0}=x^{*}$ to obtain $L^{*} \equiv$ $L^{*}(n, k)=\min L_{0}=\min \min L$. A third much simpler problem, for $x_{i}$ equally spaced, $x_{1}=-1, x_{n}=1$, is to find $\bar{x}$ to give $\bar{L} \equiv \bar{L}(n, k)=\min L$. For lower values of $n$, some results were obtained on $L_{0}$ and $L^{*}$ when $k=1$, and on $\bar{L}$ when $k=1$ and 2 by direct calculation from available tables of $L_{i}^{n(k)}(x)$. The relation of $L_{0}, L^{*}$ and $\bar{L}$ to equally spaced points, Chebyshev points, Chebyshev polynomials $T_{m}(x)$ for $m \leqslant n-1$, minimax solutions, and central difference formulas, considering also larger values of $n$, is indicated sketchily.
\end{abstract}

I. Introduction. One of the main problems in numerical differentiation is the loss in accuracy due to the size of the coefficients in the formulas that are used. Here, we are concerned with the differentiation of the $n$-point Lagrangian interpolation polynomial of the $(n-1)$ th degree, i.e.,

$$
f^{(k)}(x)=\sum_{i=1}^{n} L_{i}^{n(k)}(x) f\left(x_{i}\right)+R_{n}(x)
$$

where

$$
L_{i}^{n}(x)=\prod_{j=1, j \neq i}^{n}\left(x-x_{j}\right) / \prod_{j=1, j \neq i}^{n}\left(x_{i}-x_{j}\right)
$$

for $x$ and $x_{i}, i=1(1) n$, within $[-1,1]$. For a detailed discussion of the remainder

Received December 18, 1973.

AMS (MOS) subject classifications (1970). Primary 65D25; Secondary 65D05, 65G05.

Key words and phrases. Numerical differentiation, Lagrangian interpolation, optimally stable numerical differentiation, criteria for optimal stability, minimin and minimax solutions, Chebyshev points, Chebyshev polynomials, central difference formulas. 
$R_{n}(x)$, see [1, pp. 154-162] or [2] ; for optimization of $R_{n}(x)$ by the proper choice of $x_{i}$, see [3]. Here, $R_{n}(x)$ is assumed to be negligible, and we are interested in criteria to minimize $L \equiv L\left(n, k, x_{1}, \cdots, x_{n} ; x\right.$ or $\left.x_{0}\right)=\sum_{i=1}^{n} \mid L_{i}^{n(k)}\left(x\right.$ or $\left.x_{0}\right) \mid$ by proper choice of $x_{i}, i=1(1) n$. This is important when $f(x)$ is known, calculated, observed, measured, or found experimentally to a fixed number of decimals (e.g., in experimental physics, engineering, data reduction, physical chemistry, space sciences, trajectories, satellites in orbit, etc.). An explicit solution is known to the slightly related problem of finding $x_{i}$ to minimize the maximum of $L$ for variable $x$ in $[-1,1]$, namely $x_{i}=$ $-\cos [(i-1) \pi /(n-1)]$, which is independent of $k[4],[5],[6]$. However, in many practical problems, for optimal stability in numerical differentiation at any particular point $x$, we may choose several more suitable locations of points (one of which involves a mini-min instead of mini-max), which is the subject of this present note.

II. Two Criteria for Optimality. One problem, for $x$ fixed at $x_{0}$, is to determine $x_{i} \equiv x_{i}\left(x_{0}\right), i=1(1) n$, to obtain $L_{0} \equiv L\left(n, k, x_{0}\right)=\min L .\left({ }^{1}\right)$ Then another problem is to determine the $x_{0}=x^{*}$ that minimizes $L_{0}$ to $L^{*} \equiv L^{*}(n, k)=\min \min L$. Here, $\min$ $\min L$ is, of course, $\min L$ considered for the $n+1$ variables $x_{0}$ and $x_{i}$. Questions about the uniqueness of the minimizing sets of $x_{i}$ and $x_{0}$ will not be stressed here. We assume $x_{0} \geqslant 0$, since for $x_{0}<0$ we change the variable to $x^{\prime}=-x$. Since $L^{*}$ is preferable to any other $L_{0}$, to find $f^{(k)}(x)$ by (1) for any particular $x=x_{0}$, the variable is shifted to $x^{\prime}=x+x^{*}-x_{0}$, so that $x=x_{0}$ becomes $x^{\prime}=x^{*}$, and $x=x_{i}-x^{*}+x_{0}$ becomes $x^{\prime}=x_{i} \equiv x_{i}\left(x^{*}\right)$. This variable shift is permissible when $R_{n}\left(x^{*}\right)$ for the new $[-1,1]$ interval is still negligible, which is usually the case, except near the end of the range of $x$. To obtain $f^{(k)}(x)$ for a succession of different $x$ 's, each of which is shifted to become $x^{*}$, requires $f(x)$ at different sets of points corresponding to $x_{i} \equiv x_{i}\left(x^{*}\right), i=1(1) n$, after the shift. This amount of computation is a reasonable price for the utmost in accuracy in $f^{(k)}(x)$, when $f(x)$ itself is of limited accuracy but readily available through observation, measurement or interpolation to that full limited accuracy. $\left({ }^{2}\right)$ When $x_{0}$ cannot be shifted to $x^{*}$, such as when (a) the entire range of $x$ is too small, (b) $x_{0}$ is near or at the end of the range, or (c) a shift pushes the new interval $[-1,1]$ to where it either includes or is too near a singularity of $f(x)$, then $L_{0}$ must suffice.

(1) T. J. Rivlin discovered a fundamental error in a paper of D. L. Berman [7] that gave, rather cryptically, a purportedly complete solution based upon the work of W. Markoff [8]. In several private communications, Rivlin verified the first of five parts of Berman's crucial Theorem 2 on pp. 13-14, discredited the other parts (giving also a counterexample), and subsequently stated that he was preparing for publication his own complete solution in which the $x_{i}$ 's cannot always be given explicitly.

(2) In connection with interpolation for $f(x)$, the number of points needed (which might lie in an interval different from $[-1,1]$ ) may differ from $n$. Thus, in the extreme case where $f(x)$ is a critical table where no interpolation is necessary (in other words, $n=1$ for interpolation), we still need $n>1$ for numerical differentiation. 
A somewhat simpler looking, but entirely equivalent, formulation of the $L^{*}$ problem is to solve for $x^{*}$ and $x_{i}, i=1(1) n$, in $[-1,1]$, where

$$
\begin{array}{rlrl}
\sum_{i=1}^{n} A_{i} x_{i}^{j} & =0, & j & =0(1) k-1, \\
& =j(j-1) \cdots(j-k+1) x^{* j-k}, j & =k(1) n-1,
\end{array}
$$

and

$$
\sum_{i=1}^{n}\left|A_{i}\right|=\text { minimum }
$$

In (3) and (3'), and also in (3a) and (3'a) below, $A_{i} \equiv L_{i}^{n(k)}\left(x^{*}\right) \cdot\left({ }^{3}\right)$ Since the translation $x^{\prime}=x-x^{*}, x_{i}^{\prime}=x_{i}-x^{*}$, leaves $L_{i}^{n(k)}(x)$ unchanged, (3) and (3') may be reformulated so that we always have $x^{\prime *}=0$, and we must find $x_{i}^{\prime}$ and $X$, where $x_{i}^{\prime}$ is in the variable interval $[X, X+2]$ where $X$ is in $[-2,0]$, so that

and

$$
\begin{aligned}
\sum_{i=1}^{n} A_{i} x_{i}^{\prime j} & =k !, & j=k, \\
& =0, & j \neq k,
\end{aligned}
$$

$$
\sum_{i=1}^{n}\left|A_{i}\right|=\text { minimum. }
$$

III. Some Scattered Results. For $L_{0}$, at the endpoint $x_{0}=1$, for every $n$ and $k, x_{i}=-\cos [(i-1) \pi /(n-1)], i=1(1) n$, which is independent of $k$, and

$$
L_{0}=T_{n-1}^{(k)}(1)=\overline{n-1}^{2}\left(\overline{n-1^{2}}-1^{2}\right) \cdots\left(\overline{n-1^{2}}-\overline{k-1}{ }^{2}\right) / 1 \cdot 3 \cdots(2 k-1) .
$$

Proof. Applying (1) to $T_{n-1}^{(k)}(1)$, since $\left|T_{n-1}(x)\right| \leqslant 1, T_{n-1}^{(k)}(1)$ is a lower bound for $L$ at $x_{0}=1$, for any $x_{i}$, i.e., $T_{n-1}^{(k)}(1) \leqslant L_{0}$. But $T_{n-1}^{(k)}(1)$ is also an upper bound for $L$ when $x_{i}=-\cos [(i-1) \pi /(n-1)], i=1(1) n$, for any $x_{0}$ in $[-1,1]$, by the minimax property [4], [5], [6], i.e., $L_{0} \leqslant L$ for $x_{i}=$ $-\cos [(i-1) \pi /(n-1)]$, at $x_{0}=1, \leqslant T_{n-1}^{(k)}(1)$. The last two statements together imply $L_{0}=T_{n-1}^{(k)}(1), x_{i}=-\cos [(i-1) \pi /(n-1)], i=1(1) n$. Q.E.D. That $x_{i}=$ $-\cos [(i-1) \pi /(n-1)]$ are also the points for optimally stable extrapolatory differentiation, as well as extrapolation, when $k \geqslant 0, x_{0}>1$, follows from

$$
\sum_{i=1}^{n}\left|L_{i}^{n(k)}\left(x_{0}\right)\right|=\left|\sum_{i=1}^{n}(-1)^{i} L_{i}^{n(k)}\left(x_{0}\right)\right|=T_{n-1}^{(k)}\left(x_{0}\right)=L_{0},
$$

the last because $T_{n-1}^{(k)}\left(x_{0}\right)$ is a lower bound for every $L$.

The preceding results for $x$ within $[1, \infty]$ are special cases of Berman's Theorem 2, part 1, [7] which states that for $x_{i}=-\cos [(i-1) \pi /(n-1)]$ and $x_{0}$

$\left.{ }^{3}\right)$ Elsewhere $A_{i}$ denotes $L_{i}^{n(k)}\left(x_{0}\right)$ for whatever $x_{0}$ is under discussion. 
within any of the intervals $\left(-\infty, \xi_{1}\right),\left(\eta_{j}, \xi_{j+1}\right), j=1(1) n-2-k,\left(\eta_{n-1-k}, \infty\right)$, where $L_{1}^{n(k)}\left(\eta_{i}\right)=0, L_{n}^{n(k)}\left(\xi_{i}\right)=0, i=1(1) n-1-k$, we have $L=L_{0}=$ $\left|T_{n-1}^{(k)}\left(x_{0}\right)\right|$. From the interlacing of the $\xi_{j}$ with the $\eta_{j}, \xi_{j}<\eta_{j}$, it can be shown that for $n-k$ odd, the interval $\left(\eta_{(n-1-k) / 2}, \xi_{(n+1-k) / 2}\right)$ includes 0 , so that at $x_{0}=0, L=L_{0}=\left|T_{n-1}^{(k)}(0)\right|$. In particular, $x_{i}=-\cos [(i-1) \pi /(n-1)]$ gives $L_{0}$, when $k=1$, for $x_{0}$ within $[1 / 2,1]$ when $n=3$, within $[0,0.1076]$ or $[0.7743,1]$ when $n=4$, and within $[0.3223,0.4446]$ or $[0.8723,1]$ when $n=5$, and when $k=2$, for $x_{0}$ within $[1 / 3,1]$ when $n=4$, and within $[0,0.1319]$ or $[0.6319,1]$ when $n=5$.

For $L_{0}$, at $x_{0}=0, k=1$, a lower bound is $n-2(n-1)$ for odd (even) $n$, since $\left|T_{m}^{\prime}(0)\right|=m$ when $m$ is odd. As will be shown further on, this bound (seen above to be assumed for every even $n$ ) is assumed also for $n=3$ and 5 , is approached closely for small odd $n>5$, and to within a factor of $2 \frac{1}{2}$ for odd $n \sim 101$. For general $k, L_{0} \geqslant(=) k ! \cdot \mid$ coefficient of $x^{k} \mid$ in $T_{n-2}(x)\left(T_{n-1}(x)\right)$ for $n$ and $k$ of the same (opposite) narity.

For $k=1, n=3,4$ and 5, we may note also the following:

For $L^{*}, k=1, n=2: x^{*}=$ any point in $[-1,1], x_{1}=-1, x_{2}=1, A_{1}=$ $-1 / 2, A_{2}=1 / 2, L^{*}=1$. For $L^{*}, k=1, n=3: x^{*}=0, x_{1}=-1, x_{2}=$ any $\neq-1$ or $1, x_{3}=1, A_{1}=-1 / 2, A_{2}=0, A_{3}=1 / 2, L^{*}=1$. That we cannot improve $L^{*}=1$, for $n=2$ or 3 , is seen from $f(x)=x$, where for any choice of $x_{0}, x_{i}$ within $[-1,1]$,

$$
1=\left|f^{\prime}\left(x_{0}\right)\right|=\left|\sum_{i=1}^{n} L_{i}^{n^{\prime}}\left(x_{0}\right) x_{i}\right| \leqslant \sum_{i=1}^{n}\left|L_{i}^{n^{\prime}}\left(x_{0}\right)\right| .
$$

This inequality is an illustration of the following general property that gives lower bounds to $L^{*}$, namely, $L^{*} \geqslant \max \left|P_{m}^{(k)}\left(x^{*}\right)\right|$ of all polynomials $P_{m}(x)$ of degree $m \leqslant n-1$, where $\left|P_{m}(x)\right| \leqslant 1$ for $x$ in $[-1,1]$. Thus far we have used only the Chebyshev polynomials $T_{m}(x)$ for $P_{m}(x)$.

For $n=3$, any $x_{0},\left(x_{1}, x_{2}, x_{3}\right)=(-1,0,1), A_{1}=x_{0}-1 / 2, A_{2}=-2 x_{0}, A_{3}=$ $1 / 2+x_{0}$. When $x_{0} \geqslant 1 / 2, L=4 x_{0} \geqslant L_{0}$ must be $L_{0}$, since for $T_{2}(x)=2 x^{2}-1, T_{2}^{\prime}\left(x_{0}\right)=$ $4 x_{0} \leqslant L_{0} \cdot\left({ }^{4}\right)$ When $x_{0}<1 / 2, L=1+2 x_{0}>4 x_{0}$, which tells us no more than the already known $L_{0} \geqslant 1$ for $x_{0}$ in $[0,1 / 4]$ and $L_{0} \geqslant 4 x_{0}$ for $x_{0}$ in $[1 / 4,1 / 2]$.

For $n=4, x_{0}=0, L_{0}=3$, the $x_{i}$ being the Chebyshev points $-\cos [(i-1) \pi / 3], i=1(1) 4$, or $(-1,-1 / 2,1 / 2,1)$ for which the corresponding $A_{i}$ are $(1 / 6,-4 / 3,4 / 3,-1 / 6)$. That 3 is best is seen from $T_{3}(x)=4 x^{3}-3 x$, and $\left|T_{3}^{\prime}(0)\right|=3 .\left(^{5}\right)$ This is an illustration of where, for $x_{0}=0$, Chebyshev spacing for $x_{i}$ is better than equal spacing $\left({ }^{6}\right)$ for which $L=3.5$. However, $L^{*}$ for $n=4$ is

(4) Cf. same result in 4 th preceding paragraph.

(5) Cf. same result in 5 th preceding paragraph.

(6) In "equal spacing" it is always understood that $x_{1}=-1$ and $x_{n}=1$. 
less than 3 , because for equal spacing of $x_{i}, L=2.16 \ldots$ for $x_{0}=0.48$ (see Schedule I). That 2.16 is within $12.5 \%$ of $L_{0}$ for $x_{0}=0.48$ follows from $L_{0} \geqslant T_{2}^{\prime}(0.48)=1.92$. From $T_{2}(x)$ and $T_{3}(x)$ it is also apparent that any improvement over 2.16 for $L^{*}$ must take place before $T_{2}^{\prime}\left(x_{0}\right)=4 x_{0}=2.16$ and after $\left|T_{3}^{\prime}\left(x_{0}\right)\right|=\left|3-12 x_{0}^{2}\right|=2.16$, or (approximately) $0.265 \leqslant x_{0} \leqslant 0.54$.

For $n=5, x_{0}=0, L_{0}=3$, which is attained for equally spaced $x_{i}$, i.e., $(-1,-1 / 2,0,1 / 2,1)$, and $A_{i}=(1 / 6,-4 / 3,0,4 / 3,-1 / 6)$, and that no improvement is possible is seen again from $\left|T_{3}{ }^{\prime}(0)\right|=3$. Here Chebyshev spacing, $x_{i}=$ $-\cos [(i-1) \pi / 4], i=1(1) 5$, for $x_{0}=0$, gives the appreciably larger $L=3.83$; trying the other Chebyshev points $x_{i}=-\cos [(2 i-1) \pi / 10], i=1(1) 5$, which do not include the endpoints of $[-1,1]$, we get, surprisingly enough, $L=3.40$.

IV. First Derivative at Midpoint, $n$ Large. Around $x_{0}=1$, as $n$ increases, $L$ becomes prohibitively large for $x_{i}$ equally spaced. By comparison, when the $x_{i}$ are either of the Chebyshev points $-\cos [(i-1) \pi /(n-1)]$ or $-\cos [(2 i-1) \pi / 2 n]$, $L$ is tremendously reduced. Thus, for $n=100, k=1$, the reduction is from the neighborhood of $10^{28}$ or more, to around $10^{4}$. We have just noted, in the preceding paragraph, for $n=5, k=1, x_{0}=0$, that Chebyshev spacing for $x_{i}$ is not quite so good as equal spacing. Then for very large odd $n$, say, $n=101$, for which Chebyshev spacing is so vastly better at the endpoint, it is also of interest to make a similar comparison for $k=1$, at $x_{0}=0$. We calculate $L$ by finding $L_{i}^{101 '}(0)$ as the coefficient of $x$ in $L_{i}^{101}(x)$, as given in (2).

(a) Equal spacing, $x_{i}=-1+(i-1) / 50$ : Taking into account the oddness of $\Pi_{i=1}^{101}\left(x-x_{j}\right)$, and $L_{102-i}^{101 \prime}(0)=-L_{i}^{101 '}(0)$, we find that $L=2 \times 50 \times 50 !^{2} \times$ $\sum_{i=1}^{50} 1 / i(i+50) !(50-i)$ !, which is around 225. (See also VI, 2nd paragraph.)

(b) Chebyshev spacing, $x_{i}=-\cos [(i-1) \pi / 100]$ : Here

$$
\left|\prod_{j=1, j \neq i}^{101}\left(x-x_{i}\right)\right|=\left|\left(x^{2}-1\right) U_{99}(x) / 2^{99}\left(x-x_{i}\right)\right|
$$

where $U_{n}(x)=\sin (n+1) \theta / \sin \theta, x=\cos \theta$, is the Chebyshev polynomial of the second kind $\left[9\right.$, p. 156] , and $\left|1 / \prod_{j=1, j \neq i}^{101}\left(x_{i}-x_{j}\right)\right|=2^{99} / 100$ for $i \neq 1,101$, $=2^{98} / 100$ for $i=1,101\left[9\right.$, p. 157]. The coefficient of $x$ in $U_{99}(x)$ is -100 , so that after division and summation, noting that $L_{51}^{101 '}(0)=0$, we get

$$
L=2 \cdot 100 \cdot \frac{2^{99}}{100} \cdot \frac{1}{2^{99}} \sum_{i=1}^{50} 1 / \cos [(i-1) \pi / 100]
$$

$\left(i=1\right.$ term in $\Sigma^{\prime}$ is halved $)=2 \Sigma_{i=1}^{\prime 50} \sec [(i-1) \pi / 100]$, which is close to 300 .

(c) Chebyshev spacing, $x_{i}=-\cos [(2 i-1) \pi / 202]$ : The absolute value of the coefficient of $x$ in the numerator of (2) is $101 / 2^{100}\left|x_{i}\right|$, and 


$$
\left|1 / \prod_{j=1}^{101}\left(x_{i}-x_{j}\right)\right|=2^{100} \sin [(2 i-1) \pi / 202] / 101
$$

so that $L=2 \sum_{i=1}^{50} \tan [(2 i-1) \pi / 202]$, which is approximately 260 .

Thus, neither of the Chebyshev spacings is as good as equal spacing, for which $L \sim 225$, if not actually $L_{0}$, is at worst less than $2 \frac{1}{2}$ times $L_{0} \geqslant 99=L_{0}$ for $n=100$. Comparison of this $L \sim 225 \geqslant L^{*}$ with the Chebyshev point minimax of $10^{4}$ shows that $\min \min / \min \max <1 / 44 .\left({ }^{7}\right)$

V. Optimality for Equal Spacing, with Schedules. A drastic but practical simplification of the $L^{*}$ problem, which reduces the number of variables from $n+1$ to just one, is to specify the $x_{i}, i=1(1) n$, to be equally spaced, and to find the point $x=\bar{x}$ giving $\min L=\bar{L}$. There is evidence throughout this article, for both smaller and larger $n$, to indicate that $\bar{L}$ may not be too far from $L^{*}$ (e.g., in the preceding discussion for $n=101, k=1$, at $x_{0}=0$, where $L$ cannot be reduced by more than $56 \%$ even by varying all 101 points $x_{i}$, but where the shift of $x_{0}$ from endpoint to center reduces $L$, substantially for Chebyshev spacing, but spectacularly for equal spacing of the $x_{i}$ 's). Answers to the $\bar{L}$ problem, for $k=1$ and 2 , for the lower values of $n$, were found readily with the aid of two published tables which give (in a slightly different notation) $L_{i}^{n(k)}(x)$ as functions of $p=\left(x-x_{[(n+1) / 2]}\right) / h\left({ }^{8}\right)$ and $h=$ the tabular interval $x_{i+1}-x_{i}[10],[11]$.

In Schedule I, we give for $k=1$ and $n=3(1) 7, \bar{x}, L_{i}^{n \prime}(\bar{x})$ for $x_{i}=-1+$ $2(i-1) /(n-1), i=1(1) n, \bar{L}$, and for comparison, $L_{1}=$ the largest $L$, which is at $x=1$. These values were obtained by a direct calculation from [10], $\bar{x}$ given exactly (approximately) for $n$ odd (even). The $L_{i}^{n \prime}(\bar{x})$ (as well as $L_{i}^{n \prime \prime}(\bar{x})$ in Schedule II) is given exactly for the argument $\bar{x}$, even where that $\bar{x}$ is an approximate value for the true $\bar{x}$.

It is of interest to note in Schedule $I$ that $\bar{x}$ is at 0 when $n$ is odd, but considerably away from 0 when $n$ is even. In fact, at $x=0, L$ for $n=4$ and 6 is 3.5 and 6.21 , respectively, which is appreciably larger than $\bar{L}$. Since $n=4$ is the least $n$ for which $L^{*}$ is not yet known, an attempt was made (just by hand calculation) to improve the $\bar{L}=2.16$ by varying the equally spaced locations of the $x_{i}$ 's, but without any success. For $n=5$, if there should happen to be an $L^{*}<$

(7) A similar comparison, $n=101, x_{0}=0, x_{i}$ equally spaced, but for $k=2$, for which $L$ is approximately $2.2 \cdot 10^{4}$, may be made with the Chebyshev minimax of $10^{4}\left(10^{4}-1\right) / 3$. But knowing here that for $x_{i}=-\cos [(i-1) \pi / 100], L=L_{0}=\left|T_{100}^{\prime \prime}(0)\right|=10^{4}$, we may infer that $\min \min / \min \max <(1 / 3) \cdot 10^{-3}$.

$\left.{ }^{8}\right)$ Just here, $[\cdots]$ denotes the nearest integer. 
Schedule I

\begin{tabular}{|c|c|c|c|c|c|c|}
\hline$n$ & $\bar{x}$ & $i$ & $x_{i}$ & $L_{i}^{n^{\prime}}(\bar{x})$ & $\bar{L}$ & $L_{1}$ \\
\hline 3 & 0 & $\begin{array}{l}1 \\
2 \\
3\end{array}$ & $\begin{array}{c}-1 \\
0 \\
1\end{array}$ & $\begin{array}{c}-1 / 2 \\
0 \\
1 / 2\end{array}$ & 1 & 4 \\
\hline 4 & 0.48 & $\begin{array}{l}1 \\
2 \\
3 \\
4 \\
\end{array}$ & $\begin{array}{l}-1 \\
-1 / 3 \\
1 / 3 \\
1 \\
\end{array}$ & $\begin{array}{r}0.2137 \\
-1.0611 \\
-0.0189 \\
0.8663 \\
\end{array}$ & 2.16 & 10 \\
\hline 5 & 0 & $\begin{array}{l}1 \\
2 \\
3 \\
4 \\
5 \\
\end{array}$ & $\begin{array}{c}-1 \\
-1 / 2 \\
0 \\
1 / 2 \\
1 \\
\end{array}$ & $\begin{array}{c}1 / 6 \\
-4 / 3 \\
0 \\
4 / 3 \\
-1 / 6 \\
\end{array}$ & 3 & $21.33 \ldots$ \\
\hline 6 & 0.252 & $\begin{array}{l}1 \\
2 \\
3 \\
4 \\
5 \\
6\end{array}$ & $\begin{array}{c}-1 \\
-3 / 5 \\
-1 / 5 \\
1 / 5 \\
3 / 5 \\
1\end{array}$ & $\begin{array}{r}-3.74792805 / 48 \\
26.97658025 / 48 \\
-95.84104050 / 48 \\
-1.25707950 / 48 \\
80.87059975 / 48 \\
-7.00113195 / 48\end{array}$ & 4.494 & $42.66 \ldots$ \\
\hline 7 & 0 & $\begin{array}{l}1 \\
2 \\
3 \\
4 \\
5 \\
6 \\
7\end{array}$ & $\begin{array}{l}-1 \\
-2 / 3 \\
-1 / 3 \\
0 \\
1 / 3 \\
2 / 3 \\
1\end{array}$ & $\begin{array}{c}-1 / 20 \\
9 / 20 \\
-9 / 4 \\
0 \\
9 / 4 \\
-9 / 20 \\
1 / 20\end{array}$ & 5.5 & 83.2 \\
\hline
\end{tabular}

$\bar{L}=3$, it would be for $x_{i}$ unequally spaced, and at $x^{*} \neq 0$ because (as shown before) $L_{0}$, at $x_{0}=0$, is also 3 .

In Schedule II, we give, for $k=2$ and $n=4(1) 9, \bar{x}, L_{i}^{n \prime \prime}(\bar{x}), \bar{L}$ and $L_{1}$, all readily calculated from [11]. The results for $n=4$ are exact (and infinite in number), while $\bar{x}$ for $n=5(1) 9$ is given approximately, more roughly for $n=8$ and 9 , where the tabular interval for $p$ in [11] is 0.1 instead of 0.01 for $n=5(1) 7$. There is no problem for $n=3$, since for any $x_{0}, L_{1}^{3 \prime \prime}\left(x_{0}\right)=L_{3}^{3 \prime \prime}\left(x_{0}\right)=1, L_{2}^{3 \prime \prime}\left(x_{0}\right)=-2$, and $L=\bar{L}=4\left(=L_{0}=L^{*}\right.$ from $\left.T_{2}^{\prime \prime}(x)=4\right)$. 
Schedule II

\begin{tabular}{|c|c|c|c|c|c|c|}
\hline$n$ & $\bar{x}$ & $i$ & $x_{i}$ & $L_{i}^{n^{\prime \prime}}(\bar{x})$ & $\bar{L}$ & $L_{1}$ \\
\hline 4 & $\begin{array}{l}\text { any } x_{0} \text { in } \\
{[0,1 / 9]}\end{array}$ & $\begin{array}{l}1 \\
2 \\
3 \\
4\end{array}$ & $\begin{array}{l}-1 \\
-1 / 3 \\
1 / 3 \\
1 \\
\end{array}$ & $\begin{array}{l}9\left(1-3 x_{0}\right) / 8 \\
9\left(9 x_{0}-1\right) / 8 \\
9\left(-9 x_{0}-1\right) / 8 \\
9\left(3 x_{0}+1\right) / 8 \\
\end{array}$ & 4.5 & 27 \\
\hline 5 & 0.455 & $\begin{array}{l}1 \\
2 \\
3 \\
4 \\
5\end{array}$ & $\begin{array}{l}-1 \\
-1 / 2 \\
0 \\
1 / 2 \\
1 \\
\end{array}$ & $\begin{array}{l}2(-0.7457) / 3 \\
2(3.5228) / 3 \\
2(-0.0942) / 3 \\
2(-7.3972) / 3 \\
2(4.7143) / 3\end{array}$ & 10.9828 & $106.66 \ldots$ \\
\hline 6 & 0.064 & $\begin{array}{l}1 \\
2 \\
3 \\
4 \\
5\end{array}$ & $\begin{array}{l}-1 \\
-3 / 5 \\
-1 / 5 \\
1 / 5 \\
3 / 5 \\
1 \\
\end{array}$ & $\begin{array}{l}-5.8837 / 12 \\
40.2535 / 12 \\
-1.6770 / 12 \\
-102.6530 / 12 \\
78.7415 / 12 \\
-8.7813 / 12 \\
\end{array}$ & 19.8325 & $333.33 \ldots$ \\
\hline 7 & 0.27 & $\begin{array}{l}1 \\
2 \\
3 \\
4 \\
5 \\
6 \\
7\end{array}$ & $\begin{array}{l}-1 \\
-2 / 3 \\
-1 / 3 \\
0 \\
1 / 3 \\
2 / 3 \\
1 \\
\end{array}$ & $\begin{array}{l}0.28201945375 \\
-2.10932822250 \\
6.58379930625 \\
-0.17230407500 \\
-13.12289319375 \\
9.28202577750 \\
-0.74331904625 \\
\end{array}$ & 32.296 & 918.4 \\
\hline 8 & $1 / 35$ & $\begin{array}{l}1 \\
2 \\
3 \\
4 \\
5 \\
6 \\
7 \\
7 \\
8\end{array}$ & $\begin{array}{l}-1 \\
-5 / 7 \\
-3 / 7 \\
-1 / 7 \\
1 / 7 \\
3 / 7 \\
5 / 7 \\
1\end{array}$ & $\begin{array}{l}49(0.044692) / 9 \\
49(-0.415944) / 9 \\
49(1.798632) / 9 \\
49(-0.725720) / 9 \\
49(-2.928780) / 9 \\
49(2.704968) / 9 \\
49(-0.531056) / 9 \\
49(0.053208) / 9\end{array}$ & 50.105 & 2348 \\
\hline 9 & $1 / 5$ & $\begin{array}{l}1 \\
2 \\
3 \\
4 \\
5 \\
6 \\
7 \\
8 \\
9 \\
\end{array}$ & $\begin{array}{l}-1 \\
-3 / 4 \\
-1 / 2 \\
-1 / 4 \\
0 \\
1 / 4 \\
1 / 2 \\
3 / 4 \\
1 \\
\end{array}$ & $\begin{array}{l}-6.8860544 / 63 \\
66.4031232 / 63 \\
-288.6331392 / 63 \\
710.8562944 / 63 \\
179.6901120 / 63 \\
-1775.5508736 / 63 \\
1275.7900288 / 63 \\
-175.9039488 / 63 \\
14.2344576 / 63 \\
\end{array}$ & 71.33 & 5721 \\
\hline
\end{tabular}


We may note, from Schedule II, that for $n=4, \bar{L}=4 \frac{1}{2}$ cannot be too far from $L^{*} \geqslant 4$. For $n=5, \bar{L}=10.9828$ is very close to $L_{0}$ for $x_{0}=\bar{x}=0.455$, since $L_{0} \geqslant T_{3}^{\prime \prime}(0.455)=10.92$. Also, for $L^{*} \leqslant \bar{L}$, the $x^{*}$ must lie in the interval $[0.228,0.458]$, which is apparent from $T_{3}^{\prime \prime}(x)=24 x>10.9828$ when $x>0.458$ and $\left|T_{4}^{\prime \prime}(x)\right|=\left|96 x^{2}-16\right|>10.9828$ when $x<0.228$. Comparing $\bar{x}$ for $k=1$ with $\bar{x}$ for $k=2$, the schedules show the latter close to 0 , for $n$ even (not exactly at 0 as for $k=1, n$ odd), and close to $\bar{x}$ for $k=1$ in the $(n-1)$-point formula, for $n$ odd.

VI. More Points, Higher Derivatives. To find an approximate $\bar{x}$ and $\bar{L}$ for $k$ or $n$ outside the range of Schedules I and II, we may refer to the tables in [12] which give the exact values of $L_{i}^{n(k)}\left(x_{j}\right)$ (i.e., only at tabular points), for $k=1(1) n-1$, $n=3(1) 7$, and $k=1(1) 4, n=9,11$. The smallest $L \sim \bar{L}$ is not at the most central value of $x_{i}$ for these values of $k$ and $n$ in [12] which are not included in Schedules I and II: $k=4, n=7, \bar{x} \sim x_{5}=1 / 3 ; k=4, n=9, \bar{x} \sim x_{6}=1 / 4 ; k=2, n=11$, $\bar{x} \sim x_{8}=2 / 5 ; k=4, n=11, \bar{x} \sim x_{7}=1 / 5$.

It is interesting to note from [12] that for $k=1, n=9$ and $11, x_{0}=0$, we obtain $L=8.33$ and 11.42 , which are within $20 \%$ and $27 \%$ of $L_{0} \geqslant\left|T_{7}^{\prime}(0)\right|=7$ and $T_{9}^{\prime}(0)=9$ respectively. For odd $n=2 m+1, k=1, x_{0}=0$, it may also be shown from (5) below and [13] that $L=m \sum_{i=1}^{m} 1 / i$, so that

$$
L /(n-2)=(m /(2 m-1)) \sum_{i=1}^{m} 1 / i \geqslant L / L_{0} .
$$

From [12], for $n=11, k=2(4), L$ is around $171\left(2.48 \cdot 10^{4}\right)$ at $x_{0}=0$, and down to around $156\left(1.85 \cdot 10^{4}\right)$ at $x_{0}=1 / 5$, considerably better than the minimax $L=$ $3300\left(8.24 \cdot 10^{5}\right)$ for $x_{i}=-\cos [(i-1) \pi / 10], i=1(1) 11$; but since at $x_{0}=0$ it is known that $L_{0}=\left|L_{10}^{\prime \prime}(0)\right|=100\left(\left|L_{10}^{(4)}(0)\right|=0.96 \cdot 10^{4}\right), \min \min / \mathrm{min} \max$ may be expected to be $<1 / 33(85)$.

VII. Central Difference Formulas for Large $n$. Even though in some preceding examples, for various $n$, and the $x_{i}$ equally spaced, the $L$ would not have been improved appreciably, even if we had obtained $L=L_{0}$ at the same point $x_{0}$ by changing all the $x_{i}$, still the drop from $L$ to $L^{*}$, by varying $x_{0}$ with the $x_{i}$, could be considerably greater. However, at present, pending further specific information on $L_{0}$ and $L^{*}$, and the accompanying $x_{i}$ and $L_{i}^{n(k)}\left(x_{0}\right)$, especially for large $n$, for many practical problems we might choose $x_{0}=0, x_{i}$ equally spaced, and find that the limits to the tolerance in $L$ would not be exceeded. In employing $x_{0}=0$, $x_{i}$ equally spaced, for $n>11$, computing or using the $L_{i}^{n(k)}(0)$ becomes cumbersome, and it is more convenient to choose an odd $n=2 m+1$ and employ numerical differentiation formulas in terms of central differences $\delta_{0}^{2 r}$, for $k$ even, and mean central differences $\mu \delta_{0}^{2 r-1}$, for $k$ odd, as far as the term $r=m$ [13]. These formulas have the advantages of consisting of terms with small factors and varying with $n$ 
only in their end terms. They are given by

$$
\begin{aligned}
f^{(k)}\left(x_{0}\right) & \cong \frac{1}{h^{k}} \sum_{r=(k+1) / 2}^{m} A_{2 r-1}^{k} \mu \delta_{0}^{2 r-1}, & & k \text { odd } \\
& \cong \frac{1}{h^{k}} \sum_{r=k / 2}^{m} A_{2 r}^{k} \delta_{0}^{2 r}, & & k \text { even }
\end{aligned}
$$

where $h=x_{i+1}-x_{i}$. In our notation, after adjusting the range of $x_{i}$ to $[-1,1]$, $x_{0}$ in (4) is the central argument $x_{m+1}=0$, and the right member of (4) is identical with the right member of (1) without $R_{n}(x)$, for $x=0$. From (4), we find

$$
\begin{aligned}
L & =\frac{1}{h^{k}} \sum_{r=(k+1) / 2}^{m}\left|A_{2 r-1}^{k}\right| \cdot{ }_{2 r-1} C_{r}, & & k \text { odd }, \\
& =\frac{1}{h^{k}} \sum_{r=k / 2}^{m}\left|A_{2 r}^{k}\right| \cdot 2^{2 r}, & & k \text { even. }
\end{aligned}
$$

To prove (5), we obtain the coefficients of $f\left(x_{i}\right)$ on the right side of (4), using the coefficients of $f\left(x_{i}\right)$ in $\mu \delta_{0}^{2 r-1}$ and $\delta_{0}^{2 r}$, and taking into account the alternation with $r$ in the signs of $A_{2 r-1}^{k}$ and $A_{2 r}^{k}$.

In (4) and (5), the formulas for odd $k$ may be expected to give an $L$ that is closer to $L^{*}$ than for even $k$ (cf. Schedules I and II, and [12]). For even $k$, it appears that a better $L$ than that from (4) and (5) is had by differentiating Stirling's interpolation formula [1, pp. 67-68] and setting $x=1.8 /(n-1)$ instead of 0 , to obtain a formula for $f^{(k)}(1.8 /(n-1))$ in terms of both $\mu \delta_{0}^{2 r-1}$ and $\delta_{0}^{2 r}$.

941 Washington Avenue

Brooklyn, New York 11225

1. L. M. MILNE-THOMSON, Calculus of Finite Differences, Macmillan, London, 1933.

2. J. F. STEFFENSEN, Interpolation, Williams and Wilkins, Baltimore, Md., 1927, pp. 60-68.

3. H. E. SALZER, “Optimal points for numerical differentiation," Numer. Math., v. 2, 1960, pp. 214-227. MR 22 \# 658 .

4. D. L. BERMAN, "The solution of an extremal problem of the theory of interpolation," Dokl. Akad. Nauk SSSR, v. 87, 1952, pp. 167-170. (Russian) MR 14, 542.

5. T. J. RIVLIN, An Introduction to the Approximation of Functions, Blaisdell, Waltham, Mass., 1969, pp. 117-118. MR 40 \#3126.

6. R. J. DUFFIN \& A. C. SCHAEFFER, "A refinement of an inequality of the brothers Markoff," Trans. Amer. Math. Soc., v. 50, 1941, pp. 517-528. MR 3, 235.

7. D. L. BERMAN, "The solution of an extremal problem of the theory of interpolation," Izv. Vyšs. Učebn. Zaved. Matematika (43), v. 6, 1964, pp. 10-14. (Russian) MR 30 \#632.

8. W. MARKOFF, “ت̈ber Polynome, die in einem gegebenen Intervalle möglichst wenig von Null abweichen," Math. Ann., v. 77, 1916, pp. 213-258 (translated from the Russian, St. Petersburg Acad., 1892).

9. H. E. SALZER, "Lagrangian interpolation at the Chebyshev points $x_{n, \nu} \equiv \cos (\nu \pi / n)$, $v=0(1) n$; some unnoted advantages," Comput. J., v. 15, 1972, pp. 156-159; ibid., v. 16, 1973, pp. 190, 382. MR $47 \# 4414$. 
10. H. E. SALZER, Table of Coefficients for Obtaining the First Derivative Without Differences, Nat. Bur. Standards, Washington, D. C., Appl. Math. Ser., no. 2, 1948, 20 pp. MR 10, 69.

11. H. E. SALZER \& P. T. ROBERSON, Table of Coefficients for Obtaining the Second Derivative Without Differences, Convair-Astronautics, San Diego, Calif., 1957, 25 pp. MR 19, 689.

12. W. G. BICKLEY, “Formulae for numerical differentiation," Math. Gaz., v. 25, 1941, pp. 19-27. MR 2, 240.

13. H. E. SALZER, "Coefficients for numerical differentiation with central differences," J. Math. Phys. Mass. Inst. Tech., v. 22, 1943, pp. 115-135. MR 5, 50. 